LAWRENCE LIVERMORE N A T IO N A L LABORATORY

\section{Improved Pinhole-Apertured Point-Projection Backlighter Geometry}

B. Blue, H. F. Robey, J. F. Hansen

April 19, 2004

High Temperature Plasma Diagnostics Conference San Diego, CA, United States

April 18, 2004 through April 22, 2004 
This document was prepared as an account of work sponsored by an agency of the United States Government. Neither the United States Government nor the University of California nor any of their employees, makes any warranty, express or implied, or assumes any legal liability or responsibility for the accuracy, completeness, or usefulness of any information, apparatus, product, or process disclosed, or represents that its use would not infringe privately owned rights. Reference herein to any specific commercial product, process, or service by trade name, trademark, manufacturer, or otherwise, does not necessarily constitute or imply its endorsement, recommendation, or favoring by the United States Government or the University of California. The views and opinions of authors expressed herein do not necessarily state or reflect those of the United States Government or the University of California, and shall not be used for advertising or product endorsement purposes. 
UCRL-CONF-203624

\section{Improved Pinhole-Apertured Point-Projection Backlighter Geometry}

B.E. Blue ${ }^{\text {a) }}$ J.F. Hansen, and H.F. Robey

Lawrence Livermore National Laboratory, Livermore, CA 94550

a)Electronic mail: blue3@1lnl.gov

Pinhole-apertured point-projection $\mathrm{x}$-ray radiography is an important diagnostic technique for obtaining high resolution, high contrast, and large field-of-view images used to diagnose the hydrodynamic evolution of high energy density experiments. In this technique, a pinhole aperture is placed between a laser irradiated foil (x-ray source) and an imaging detector. In this letter, we present an improved backlighter geometry that utilizes a tilted pinhole for debris mitigation and a front-side illuminated backlighter foil for improved photon statistics.

\section{Introduction}

Pinhole-apertured point-projection x-ray radiography ${ }^{1}$ is an important tool for diagnosing the hydrodynamic evolution of high energy density experiments ${ }^{2-4}$. The technique uses an $\mathrm{x}$-ray source that is produced by illuminating a thin backlighter foil with high intensity laser beams. The x-ray source is then imaged onto a detector using a pinhole aperture. A sample to be radiographed is placed between the pinhole and the detector. This radiographic technique is advantageous to other techniques, such as area backlighters, in that it can produce a high resolution, high contrast, and large field-ofview image at a correspondingly lower laser intensity. ${ }^{5}$ 
Previous experiments that utilized area backlighters used foils that were both front and/or rear illuminated. ${ }^{6}$ It has been shown that by utilizing a front illuminated area backlighter foil, an increased photon count will be witnessed by the detector. Previous point-projection backlighter geometries have used a backlighter foil that was parallel to the pinhole substrate and separated by $\sim 500 \mu \mathrm{m}$ (fig. 1A). In these configurations, it was impractical to illuminate the front side (the side facing the pinhole substrate) of the backlighter foil due to its close proximity to the pinhole substrate. Extending the foil-topinhole separation to allow for front side illumination, in which the laser beams would strike the backlighter foil at an angle less than $60^{\circ}$, would negate the benefits of using a pinhole-assisted point-projection backlighter since the x-ray spot size needed to illuminate the target through the pinhole would need to be very large. In this paper we present a new geometry that allows the backlighter foil to be front illuminated. As shown in fig $1 \mathrm{~B}$, this geometry utilizes a tilted pinhole for debris mitigation and a backlighter foil that is perpendicular to the pinhole substrate.

\section{Experimental Setup}

The experiment was performed at the OMEGA Laser Facility. ${ }^{7}$ Five $500 \mathrm{~J}, 1 \mathrm{~ns}$, $351 \mathrm{~nm}$ laser beams were used to illuminate a $5 \mu \mathrm{m}$ thick titanium backlighter foil. The laser spot size of $500 \mu \mathrm{m}$ corresponded to an intensity of $1 \times 10^{15} \mathrm{~W} / \mathrm{cm}^{2}$. Two different pinhole geometries were tested. In the first geometry, the backlighter foil was parallel to the pinhole substrate. This setup, detailed in Fig. 1a, consisted of a $5 \mu \mathrm{m}$ titanium backlighter foil placed $400 \mu \mathrm{m}$ away from a $2.5 \mathrm{~mm}$ x $3 \mathrm{~mm}$ x $50 \mu \mathrm{m}$ tantalum pinhole. The second setup utilized the perpendicular backlighter foil design. It consisted of a 5 
$\mu \mathrm{m}$ titanium backlighter foil oriented perpendicularly to a $2.5 \mathrm{~mm}$ x $3 \mathrm{~mm}$ x $50 \mu \mathrm{m}$ tantalum pinhole. This setup is detailed in Fig. $2 b$. The pinhole apertures were made by laser cutting a $10 \mu \mathrm{m}$ hole into the tantalum substrate. The tilted pinhole apertures were drilled at a $30^{\circ}$ angle so that the axis of the aperture was oriented towards the diagnostic. Both pinholes were coated in $4 \mu \mathrm{m}$ of parylene to reduce fluorescence from the pinhole substrate.

The experimental setup was configured to measure both the imaging characteristics of both pinhole geometries and also to measure the spectral output of both backlighter foil geometries. In order to measure the imaging characteristics of the pinhole geometries, a 400-mesh gold grid placed $10 \mathrm{~mm}$ away from the pinhole was imaged $244 \mathrm{~mm}$ away onto a gated imaging detector. The magnification of 25 meant that the system resolution was limited by the geometrical size of the pinhole. The camera was filtered with 30 mils of beryllium. The spectral output of the backlighter foil was measured with the Henway spectrometer. This diagnostic provided an absolutely calibrated measurement of the photon yield between 4.5 and $10 \mathrm{keV}$.

\section{Experimental Results}

The x-ray source on the backlighter foil was the same for both geometries since the laser intensity was not varied. However, the x-ray source as seen through the pinhole to the diagnostic varied depending on whether the source was attenuated by the backlighter foil. The spectral output of the two different geometries is shown in fig. 2 . The $4.7 \mathrm{keV}$ titanium $\mathrm{He}_{\alpha}$ line is the dominant emission in both geometries. The magnitude of the $\mathrm{He}_{\alpha}$ line is smaller in the rear-illuminated geometry (dashed line) than 
the front-illuminated geometry (solid line) because the emission is attenuated by the opacity of the $5 \mu \mathrm{m}$ thick titanium backlighter foil. The other major difference between the two geometries is the lack of higher energy spectral content in the rear-illuminated geometry. This is due to the fact that the titanium backlighter foil is cutting off the spectral content above its $5 \mathrm{keV}$ K-edge.

Images of a 400-mesh grid were acquired in order to measure the imaging characteristics of both pinhole geometries. Figure 3a shows a line out from the frontilluminated (FI) geometry and fig 3b shows one from the rear-illuminated (RI) geometry. The peak exposures are the hole of the grid where the x-rays are unattenuated. The dips in exposure are the regions where the x-rays were attenuated by the grid wires. The RI geometry shows an average exposure of $0.14 \mathrm{ergs} / \mathrm{cm}^{2}$, while the FI geometry has an average exposure of $0.5 \mathrm{ergs} / \mathrm{cm}^{2}$. The FI geometry gave an $\sim 3.5$ increase in exposure while using the same laser power. Figure 4a shows the signal-to-background ratio from the front-illuminated (FI) geometry and fig. $4 \mathrm{~b}$ shows one from the rear-illuminated (RI) geometry. This ratio was made by taking the signal level shown in fig. 3 and dividing it by a line out taken along a grid wire. The line out along the grid waire was chosen since it accounts for gain variations in the MCP. The FI geometry has a SB ratio of 2-3 while the FI geometry has a 5x improved ratio of 10-15.Both geometries had a pinhole limited resolution of $7 \mu \mathrm{m}$, but the improved contrast (fig. 5) of the FI geometry resulted in a significantly improved pinhole-assisted point-projection backlighter image.

\section{Summary}


High energy density (HED) experiments require advanced x-ray imaging systems to diagnose their hydrodynamic evolution. Pinhole-assisted point-projection backlighters are an integral part of the imaging system due to their ability to generate high-resolution and high-contrast x-ray images. Future HED experiments that utilize opaque materials will require backlighters with improved contrast as compared to what is currently used. This improved backlighter geometry was conceived to address these future experimenter's needs.

\section{Acknowledgements}

The authors would like to thank J. Knauer, the staff, and operations crew at OMEGA for their assistance in performing this experiment. We would also like to thank R. Wallace and K. Loughman for their assistance in target fabrication. This work was performed under the auspices of the U.S. Department of Energy by University of California, Lawrence Livermore National Laboratory under Contract W-7405-Eng-48.

\section{References}

1. D.K. Bradley, O.L. Landen, A.B. Bullock, S.G. Glendinning, and R.E. Turner, Opt.

Lett. 27, 134 (2002).

2. H.F. Robey et al., Phys. Rev. Lett. 89, 085001 (2002).

3. R.P. Drake et al., Ap. J. 564, 896 (2002).

4. J.M. Foster et al., Phys. Plasmas 9, 2251 (2002).

5. O.L. Landen et al., Rev. Sci. Instrum. 72, 627 (2001). 
6. A.B. Bullock, O.L. Landen, and D.K. Bradley, Rev. Sci. Instrum. 72, 686 (2001).

7. J.M. Soures et al., in Proceedings of the 37th Meeting APS Division of Plasma

Physics, Louisville, KY 1996 (AIP, New York, 1996), pp. 2108-2112.
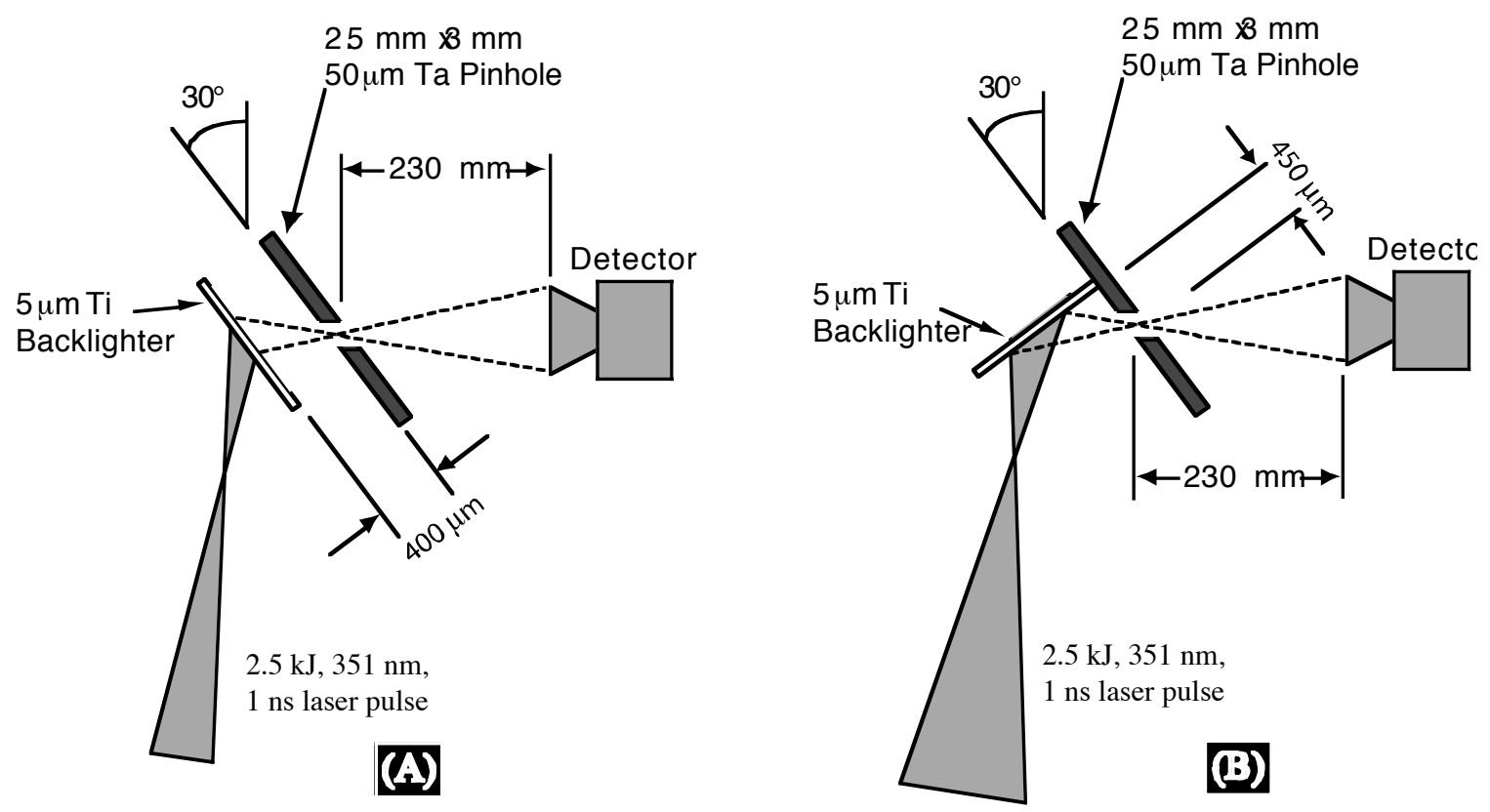

Figure 1. Pinhole-assisted point-projection backlighter geometries. The rear illuminated backlighter design is shown in part A. In this geometry, the laser strikes the rear surface (with respect to the pinhole) of the backlighter foil. The x-rays are filtered by the backlighter foil before they are transmitted through the pinhole. The front-illuminated geometry is shown in part B. This geometry is advantageous in that the laser produced $\mathrm{x}-$ rays are not attenuated by the backlighter foil before they are transmitted through the pinhole. 


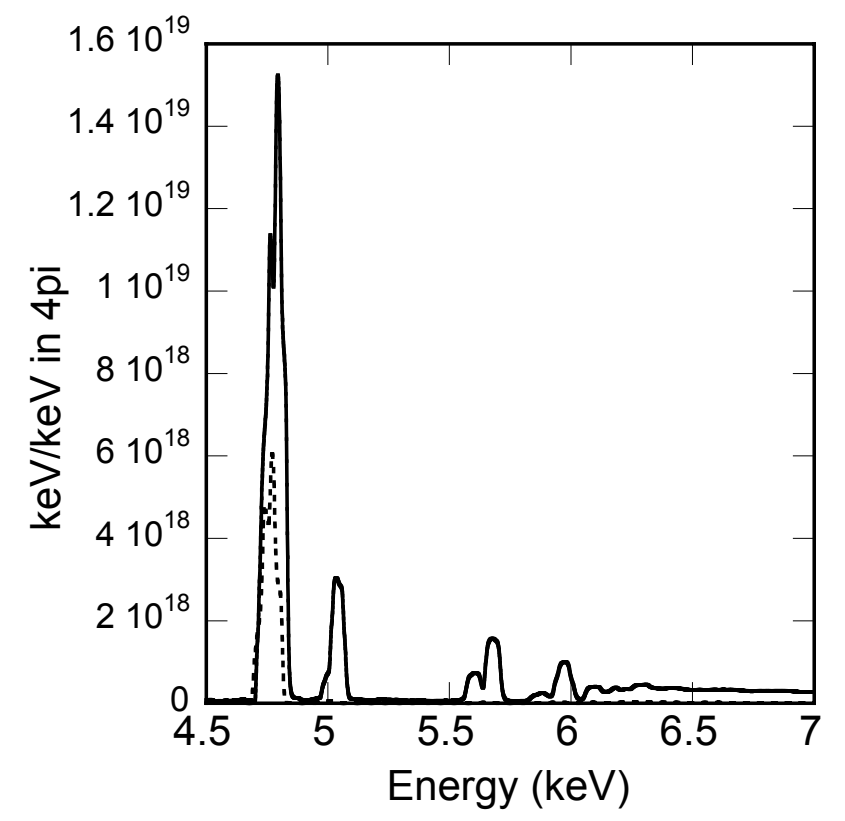

Figure 2. Spectral output of the two backlighter geometries. The front-illuminated geometry is shown as a solid line and the rear-illuminated geometry is shown as a dashed line. The front-illuminated design has a stronger $4.7 \mathrm{keV} \mathrm{He}{ }_{\alpha}$ emission line as well as higher energy lines extending up to the bound free continuum. The rear-illuminated geometry's spectrum has a weaker $\mathrm{He}_{\alpha}$ line and a much weaker higher energy content due to the transmission through the backlighter foil. 

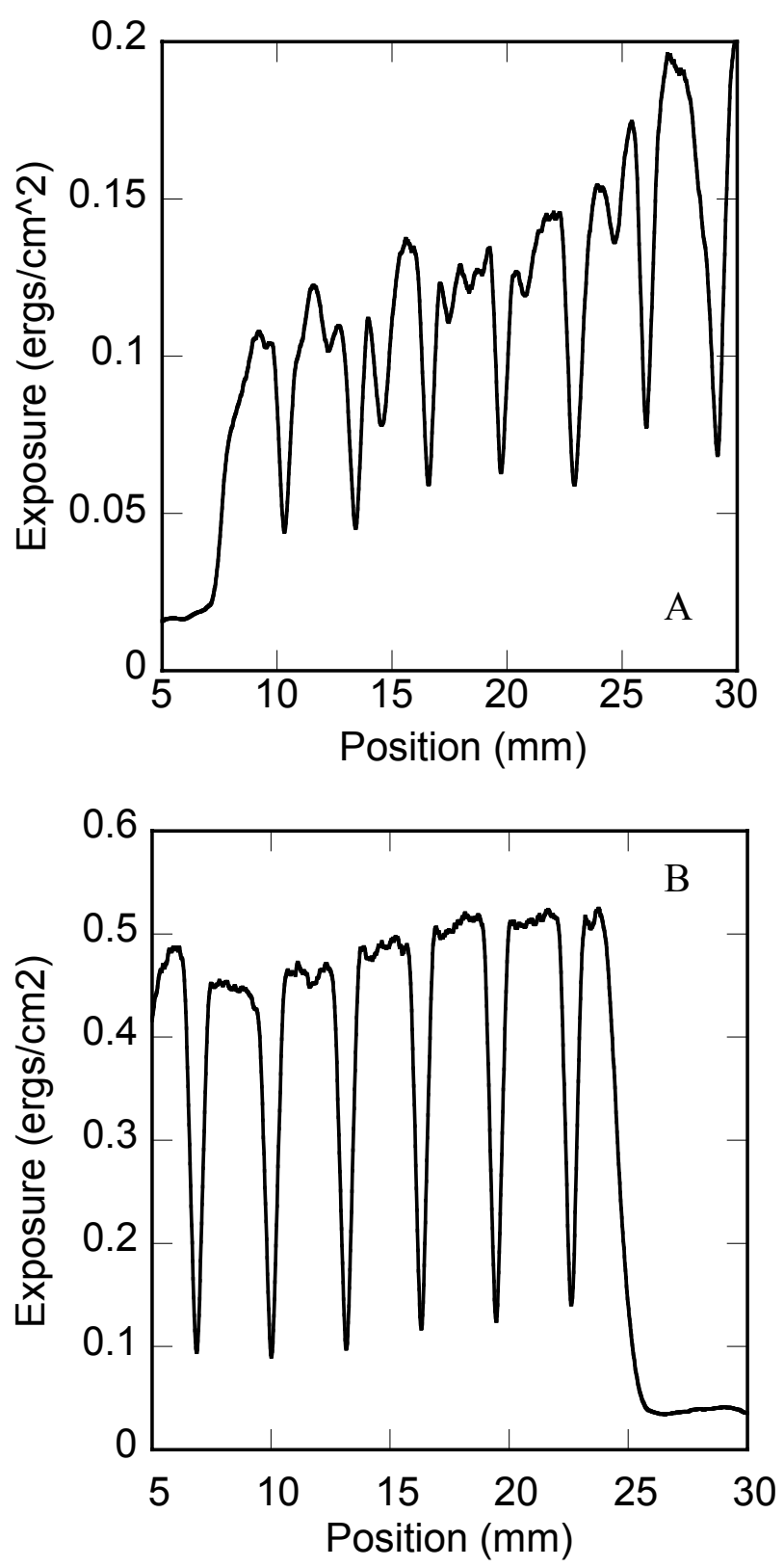

Figure 3. Line-outs of an imaged 400-mesh grid. Figure 3 a shows a line out from the front-illuminated (FI) geometry and fig 3 b shows one from the rear-illuminated (RI) geometry. The peak exposures are the hole of the grid where the x-rays are unattenuated. The dips in exposure are the regions where the x-rays were attenuated by the grid wires. The RI geometry shows an average exposure of $0.14 \mathrm{ergs} / \mathrm{cm}^{2}$, while the FI geometry has 
an average exposure of $0.5 \mathrm{ergs} / \mathrm{cm}^{2}$. The FI geometry gave an $\sim 3.5$ increase in exposure while using the same laser power.
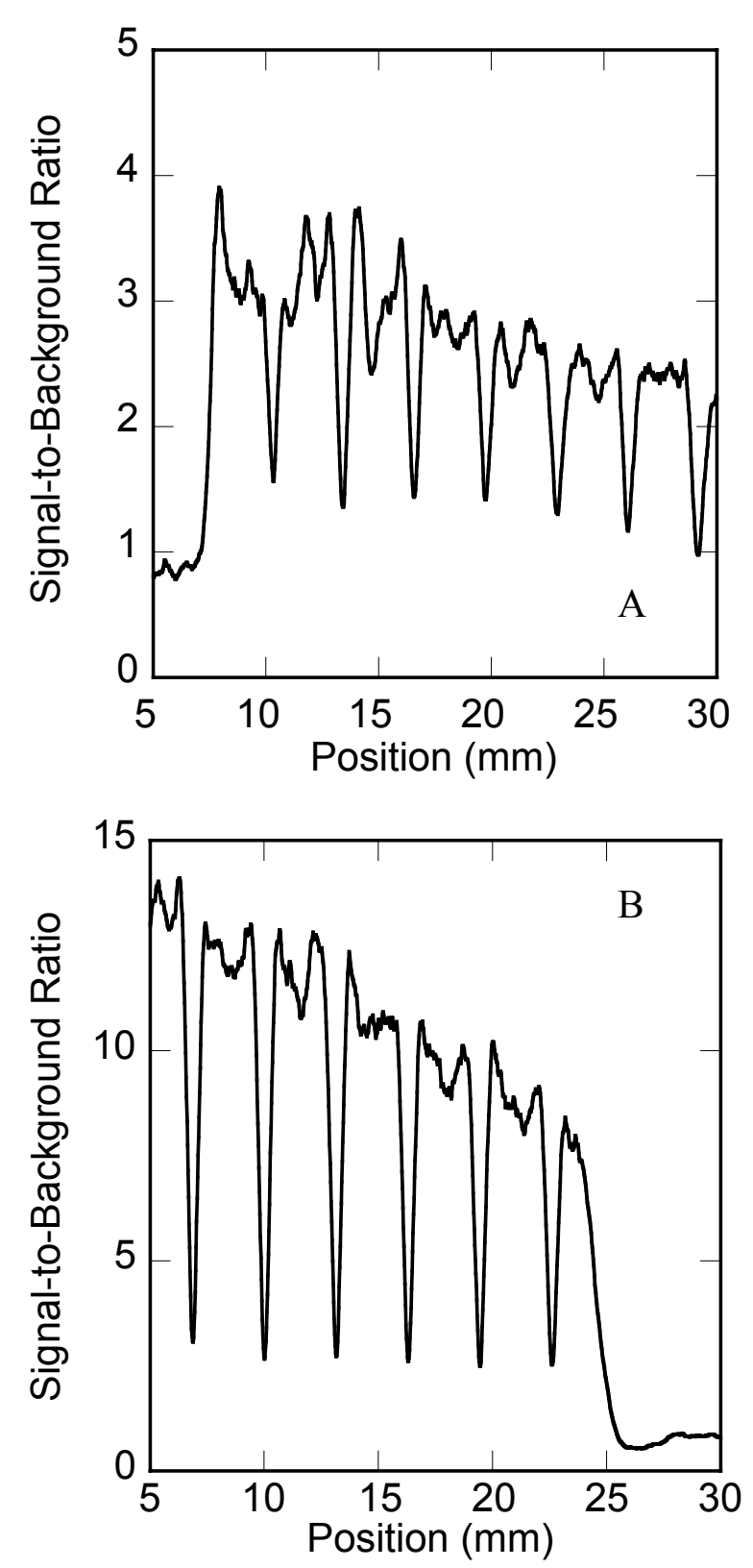
Figure 4. Signal-to-background (SB) ratio of the two backlighter geometries. Figure 4a shows the ratio from the front-illuminated (FI) geometry and fig. $4 \mathrm{~b}$ shows one from the rear-illuminated (RI) geometry. This ratio was made by taking the signal level shown in fig. 3 and dividing it by a line out taken along a grid wire. The line out along the grid waire was chosen since it accounts for gain variations in the MCP. The FI geometry has a SB ratio of 2-3 while the FI geometry has a 5x improved ratio of 10-15. 